\title{
Recursos de las Regalías Directas asignadas en el Municipio de Neiva para la Cuenca del Río Las Ceibas
}

Semillero de Investigación "GUIAS"
MARTHA CECILIA ABELLA / Directora del Proyecto

INTEGRANTES:

LUZ THAMAR FRAIJA CABRERA / Coordinadora ADADIER PERDOMO URQUINA / Fiscal JUDY KATHERIN CARDOZO ZAPATA / Tesorera MARIA MONICA MONJE CORTES / Secretaria

Universidad Surcolombiana

Facultad de Derecho Neiva, octubre de 2008

\section{RESUMEN}

En la presente investigación sobre los recursos de las regalías directas asignadas al Municipio de Neiva para la recuperación y conservación de la Cuenca del Río las Ceibas durante los años (2005-2007), se pretende verificar, si los procedimientos implementados por laAdministración Municipal para la asignación e inversión de los recursos, que por concepto de regalías, contribuyeron de manera efectiva a la recuperación y conservación de la Cuenca del Río las Ceibas, siguiendo el espíritu de la ley, la moral y las buenas costumbres.

\section{ABSTRACT}

The development of the investigation about resources of the direct royalties given to Neiva for the basin of "Las Basin River", in which Its main goal is trying to discover if the procedures that the local administration of the Neiva city, has implemented during the years (2005-2007) for development of the asignation and invertion of the resources for royalties effectively helped to conservation of the this basin.

\section{PALABRAS CLAVE}

Cuenca del Río las Ceibas, Ciudad de Neiva, Población de Neiva, acueducto, agua, regalías, protección y conservación de la Cuenca, medio ambiente.

\section{KEYWORDS}

The basin of "Las Basin River", Neiva city, water, population of the city, royalties. Aqueduct, environment, protection and conservation of the basin 
A través de la presente investigación, se da a conocer a los habitantes de la ciudad de Neiva, el estado actual de la Cuenca del Río las Ceibas, su problemática, su futuro, sus amenazas y la crisis actual de la principal fuente hídrica abastecedora del acueducto de la ciudad capital del Departamento del Huila. Para esta labor, fué necesario investigar cuáles fueron los factores que obstaculizaron ofacilitaron la debida destinación de los recursos establecidos por la ley, en beneficio de un ecosistema natural tan importante para dicha ciudad como lo es la Cuenca del Río Las Ceibas, pues como lo mencionamos antes, ésta es la principal fuente abastecedora de agua potable; de igual manera se analizaron los proyectos para establecer la inversión y según lo encontrado damos a conocer los resultados y conclusiones.

En ese sentido, esta investigación se propuso identificar y analizar si se presenta corrupción en la gestión pública, respecto de la adecuada inversión de los recursos económicos, producto de las regalías que tuvieron la destinación específica a las entidades territoriales, especialmente al municipio de Neiva; además se pretendió determinar si los propósitos para los cuales fueron destinados estos recursos tuvieron una aplicación

\section{PLANTEAMIENTO}

\section{DEL PROBLEMA}

¿Los procedimientos implementados por la administración municipal de la Ciudad de Neiva durante los años (2005-2007) para la inversión de los recursos que por concepto de regalías del presupuesto Municipal están destinados a la Recuperación y Conservación de la Cuenca del Río las Ceibas, se han enmarcado dentro de los fines' $y$ principios ${ }^{2}$ rectores de la administración pública? real respecto a su objeto, es decir, si dichos recursos se destinaron a beneficiar al ecosistema de la Cuenca del Río las Ceibas y si con ello se alcanzó la recuperación y conservación parcial de esta cuenca hidrográfica, equivalente a lo presupuestado para esta misión.

El carácter de esta investigación ha sido metodológico, crítico, analítico, con recolección de datos, y llevándolo a cabo en forma deductiva y descriptiva, pues de esta manera se estableció qué tanto se logró realizar con el dinero otorgado por parte de las regalías al Municipio de Neiva para la preservación de dicha cuenca.

No se descarta además, establecer y determinar cuál fué el proceder administrativo y de gestión pública que llevó a cabo el Municipio de Neiva, en relación a la inversión de los dineros provenientes de las regalías para la recuperación y conservación de la Cuenca del Río las Ceibas y lo más importante, es ayudar a descubrir si se ha identificado el verdadero problema que afronta esta Cuenca, y si la conducta desplegada, como quiera que se observa la adjudicación de contratos a personas Jurídicas y Naturales, han contribuido realmente a su objetivo: Recuperary Conservar la Cuenca.

\section{JUSTIFICACIÓN}

Las regalías se constituyen en la contraprestación económica que el Estado recibe por la explotación y prestación de un recurso natural no renovable, lo cual genera un beneficio económico para el Estado y para sus entidades territoriales en cuya jurisdicción, se realizan las tareas de explotación, transformación y transporte, pues son acreedoras de un derecho constitucional y de manera especial los artículos $360^{3}$ y 361 de La Carta Magna,

${ }^{1}$ ARTÍCULO 30 Ley 80 de 1993. "DE LOS FINES DE LA CONTRATACIÓN ESTATAL. LOs servidores públicos tendrán en consideración que al celebrar contratos y con la ejecución de los mismos, las entidades buscan el cumplimiento de los fines estatales, la continua y eficiente prestación de los servicios públicos y la efectividad de los derechos e intereses de los administrados que colaboran con ellas en la consecución de dichos fines.

${ }^{2}$ Artículos 24, 25, 26, yss. de la ley 80 de 1993.

${ }^{3}$ ARTICULO 360. La ley determinará las condiciones para la explotación de los recursos naturales no renovables asl como los derechos de las entidades territoriales sobre los mismos. La explotación de un recurso natural no renovable causará a favor del Estado, una contraprestación económica a título de regalía, sin perjuicio de cualquier otro derecho o compensación que se pacte. Los departamentos y municipios en cuyo territorlo se adelanten explotaciones de recursos naturales no renovables, así como los puertos marítimos y fluviales por donde se transporten dichos recursos o productos derivados de los mismos, tendrán derecho a participar en las regalías y compensaciones. 
participación directa en las regalías, definido por el legislador: ley 141 de 1994, siendo ésta el palafito normativo ${ }^{4}$ ya que se crean las instituciones encargadas del manejo del recaudo orientadas hacia los principios y fines de las regalías propuestas por la constituyente de 1991; la ley 756 de $2002,{ }^{5}$ establece la base de participación de las entidades ternitonales y su destinación.

Del $90 \%$ de los recursos de las regalias directas para los municipios productores, tiene una destinación de vital interés en términos sociales: Cubrir las necesidades básicas de la población en los sectores de salud, educación, agua potable y saneamiento básico; infortunadamente en algunos casos, el manejo de estos recursos ha sido afectado por fenómenos de dilapidación, despilfarro y en síntesis, indebida utilización, lo que de manera inevitable se traduce en el detrimento de la calidad de vida ciudadana y en un agravamiento general de la crisis social.

Con fundamento en la idea que el Río las Ceibas es considerado como un área de reserva para la preservación y conservación del medio ambiente, y que los recursos naturales, de conformidad con las normas que definen la estructura del territorio municipal, contempladas en el POT del municipio de Neiva, exigen el compromiso de todos los actores en los procesos y procedimientos que tienden a dar desarrollo y sostenibilidad al ecosistema de la Cuenca, con lo cual se impone la necesidad de la participación ciudadana para procurar el seguimiento a las actividades y de esta manera evitar que se desvíen los recursos otorgados para la protección y conservación del medio ambiente.

\section{OBJETIVOS}

\section{OBJETIVO GENERAL}

Establecer en forma objetiva los procedimientos que despliega la administración municipal de la ciudad de Neiva para llevar a cabo la asignación e inversión de los recursos que por concepto de regalías hacen parte de su presupuesto y que están destinados a la recuperación y conservación de la cuenca del río las Ceibas.

\section{OBJETIVOSESPECIFICOS}

1. Identificar los proyectos que se han orientado específicamente al estudio del ecosistema y a la protección de flora y fauna de la cuenca del río las Ceibas.

2. Establecer el objeto de los contratos y proyectos financiados con los recursos provenientes de las regalías, distribuidos por el municipio de Neiva y destinados a la preservación y conservación de la cuenca del río las Ceibas.

3. Analizar cuál ha sido el proceso de distribución e inversión de las regalías asignadas por el municipio de Neiva, para la preservación de la cuenca del Río las Ceibas, es decir se establecerá la política municipal de inversión de las regalias.

4. Establecer los procedimientos implementados por la administración municipal para llevar a cabo la inversión de los recursos provenientes de las regalías tendientes a la recuperación de la cuenca del Río las Ceibas.

5. Identificar el impacto de las actividades desarrolladas con dineros de regalias en procura de la recuperación y preservación de la cuenca del Río las Ceibas.

\section{METODOLOGÍA}

Con base en la problemática a desarrollar, se tiene en cuenta un enfoque epistemológico para la presente investigación que se orienta por una metodología cuantitativa descriptiva, puesto que iniciará con un procedimiento histórico descriptivo que comprenderá la enunciación pasada de la Cuenca, el registro, el análisis e interpretación de los datos recolectados, para luego, con base en esos resultados, obtener con precisión la situación

4Crea el Fondo Nacional de Regallas, Instaura la Comisión Nacional de Regalías, Establece el derecho del Estado por la explotación de los recursos naturales no renovables, Señala las reglas para la liquidación, distribución y utilización de regalias.

${ }^{5}$ Define porcentajes variables para el pago de las regalías de hidrocarburos, Establece el pago de una regalía adicional para los contratos revertidos a favor de la Nación después de 1994, Dispone una variación de la distribución de las regalías para los primeros 50.000 barriles promedio diario de hidrocarburos. 
actual de los procedimiento desplegados por la administración para hacer la asignación presupuestal y la inversión en la recuperación y conservación de la cuenca del Río las Ceibas.

\section{ACTIVIDADES}

\section{INVESTIGATIVAS}

1. Recopilación, revisión e identificación de la normatividad a nivel nacional, departamental y municipal que rige la materia, la doctrina y la jurisprudencia específicamente enfocada hacia las regalías directas.

2. Revisión de los contratos, informes, proyectos y resultados de las auditorías.

3. Análisis de los resultados de las distintas investigaciones adelantadas por los organismos de control competentes.

\section{RESULTADOS}

Conforme a nuestra minuciosa investigación veremos los resultados obtenidos en base a todos los centros investigados como la alcaldia de Neivay todas sus dependencias en la búsqueda de los contratos, la visita al DATMA, la CAM, Empresas Públicas de Neiva, a gran parte de la comunidad que conforman la Cuenca y a todos los principales actores que entrevistamos y visitamos para obtener los siguientes resultados:

1. Clasificación de estudios y documentos existentes sobre la cuenca del rí las ceibas.

2. Población área rural cuenca hidrográfica río las ceibas

3. Contratos analizados.

\section{PROCESAMIENTO DE DATOS Y CODIFICACIÓN}

Recolección de datos, los cuales se obtienen de la actividad material a voluntad de la administración en lo relacionado a las regalías destinadas para la preservación y recuperación de la cuenca del Río las Ceibas, los cuales se obtuvieron a través de los instrumentos diseñados para tal fin y se procesaron mediante una base de datos sistematizada, para cuantificarlos en forma debida como se hizo, lo cual permitió llegar a las conclusiones en relación con la problemática inicialmente planteada.

\section{CLASIFICACIÓN DE ESTUDIOS Y DOCUMENTOS EXISTENTES SOBRE LA CUENCA DEL RIO LAS CEIBAS.}

\section{ATRIBUTO}

CANTIDAD

Diagnósticos

15

Planes y programas

17

Documentos técnicos

Investigaciones

Informes

Consultorías

Ensayos

Monografías $^{6}$

Registros IDEAM (Todos los registros de las estaciones existentes en la cuenca, desde su fecha de instalación en 1983 hasta el año 2007)

${ }^{6}$ Universidad Surcolombiana centro de investigaciones y desarrollo científico, USCO, CIDEC, monografia de la subcuenta del río las ceibas. Recopilación de información. Neiva 1990. Universidad Surcolombiana, USCO, facultad de ingeniería ambiental, valoración económica aproximada de los costos ambientales de la cuenca del río las ceibas en el municipio de Neiva-Huila de 1999. 
Como vemos, estos son los estudios, ${ }^{7}$ realizados hasta ahora, pero para entrar más en detalle miremos el resultado que tenemos de población existente y luego entraremos a analizarla de acuerdo a los contratos y a las encuestas realizadas por el grupo de investigación.

\section{POBLACIÓN ÁREA RURAL CUENCA HIDROGRÁFICA RÍO LAS CEIBAS}

VEREDA

ALTO MOTILON

CANOAS

CEIBAS AFUERA

CHAPURO

EL CENTRO

EL VERGEL

FLORA GAITA

LA PLATA

LA PRIMAVERA

LAS NUBES

LOS CAUCHOS

MOTILON

PALESTINA

PLATANILLAL

PUEBLO NUEVO

SAN BARTOLO

SAN MIGUEL

SANTA BARBARA

SANTA LUCIA

TUQUILA

VENADITO

SANTA ROSALIA

SANTA HELENA

ALTA ROSALIA

TOTAL

\section{TOTAL HABITANTES SEGÚN \\ IDAMA CITADO POR CHARRY, 1996}

95

135

211

157

$-$

100

85

90

-

120

100

250

160

90

155

500

-

70

200

110

-

230

80

70

3008

TOTAL HABITANTES
POT 2000

101

127

283

351

568

90

100

292

79

122

86

111

170

105

133

340

130

120

-

85

141

-

75

3609

\section{TOTAL HABITANTES SISBEN ACTUAL}

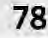

122

$80(1)$

276

160(2)

76

115

142

71

61

50

158

223

76

197

245

134

36

281

114

141(3)

86

2922

Con base en los estudios realizados, ${ }^{8}$ sobre la comunidad que habita la Cuenca, determinamos que en algunos sectores como Motilón y la Vereda La Plata, se hace urgente la evacuación de la población; pues las condiciones de estas veredas son tan graves que están declaradas como zona de alto riesgo, y por tanto determinamos que los problemas más graves que tenemos actualmente en la cuenca con base a los habitantes que la rodean y la situación actual estudiada y analizada por el grupo de trabajo teniendo en cuenta lo establecido en el trabajo de campo, lo observado en los alrededores de la Cuenca y los diálogos con los habitantes de dichas veredas son:

'Instituto Nacional de Los Recursos Naturales Renovables y del Medio Ambiente, INDERENA; Síntesis de la problemática y propuestas de acciones en cuenca del río las ceibas. Neiva, Abril de 1989; Programa de protección y manejo de la cobertura vegetal de la cuenca del río las ceibas proyecto cuenca del alto magdalena corporación autónoma regional del alto magdalena, procam, plan de comercialización agropecuaria cuenca ríos las ceibas, Yaguará y Combeima. Bogotá, julio de 1986; Instituto de investigaciones de recursos biológicos Alexander von Humbolt, programa de investigación en política y legalizaron de indicadores de seguimiento de la política de biodiversidad, programa de biología de la conservación. Bogotá, 30 de octubre de 2002.

${ }^{8}$ Contraloría Departamental del Huila. Estudio anual sobre del estado de los recursos naturales y medio ambiente del Río las Ceibas. Neiva, 2000. REVISTA SOS DE 2000; Ecosistemas estratégicos en la subcuenta del río las ceibas. Neiva 2000; informe medio ambiente, evaluación de la gestión ambiental del municipio de Neiva en la vigencia fiscal del 2003. Neiva, julio de 2004. 
- Deforestación

- Reducción en la productividad del suelo (erosión)

- Contaminación hídrica

- Quemas e incendios forestales

- Aumento de la sedimentación

- Ineficiencia gestión gubernamental

- Alteración régimen de caudales

- Demanda para uso de agua

- Uso inadecuado del suelo

- Avalanchas e inundaciones

- Alteración del paisaje

- Sequías

- Falta de conciencia ambiental

- Desprotección de laderas

- Reducción de la biodiversidad

- Procesos puntuales de degradación de la calidad del recurso hídrico, especialmente por la falta de manejo de las aguas residuales de los predios de la cuenca. Presencia de áreas geomorfológicamente inestables, agravadas por las prácticas inadecuadas de aprovechamiento y manejo de suelos, generando problemas acelerados de erosión y pérdida del recurso productivo.

- Altos aportes de sedimentos a las aguas del Río Las Ceibas y al acueducto causando interrupciones en la prestación del servicio de agua; igualmente su depositación al Río Magdalena.

- Fuerte presión humana sobre el recurso bosque, en razón de los procesos de colonización y ampliación de la frontera agrícola.

- Quemas indiscriminadas

- El equilibrio ecológico y desarrollo sostenible de la cuenca demandan mantener una extensión de terrenos en bosques que cumplan fundamentalmente una acción de regulación y protección.

- La fragmentación de los bosques de la parte alta que constituyen el corredor biológico de norte a sur del departamento.

- El fenómeno de torrencialidad que caracteriza a la cuenca en épocas de lluvia, genera avalanchas, poniendo en riesgo a los habitantes de la zona.

\section{CONTRATOS ANALIZADOS}

\subsection{Contrato No. 078 de $2005^{\circ}$}

Objeto del contrato: Construcción obras de control y protección de emergencias del río las ceibas en las orillas de los barrios Plazas Alcid, Reinaldo Matiz, Las Delicias, José Eustacio Rivera y Tenerife del Municipio de Neiva.

Estudio de convivencia y oportunidad: Se pretende proteger a un gran numero de viviendas del casco urbano que no han sido reubicadas y/o no se les ha negociado la mejora y que permanecen en amenaza de alto riesgo por las crecientes del rio las Ceibas.

\section{Contratista: José Maunicio Andrade Monje.}

Objeto: El contratista se compromete para con el Municipio a realizar por medio del sistema de precios unitarios, la obra consistente en la construcción las obras de control y protección de emergencias del río las ceibas en las orillas de los barrios Plazas Alcid, Reinaldo Matiz, Las Delicias, José Eustacio Rivera y Tenerife del Municipio de Neiva.

Valor inicial del contrato: $\quad \$ 15^{\circ} 275.000$

Valor final del contrato: $\quad \$ 15^{\prime} 210.750$

\subsection{Contrato o escritura: No.345-2005 ${ }^{10}$}

Programa:Saneamiento básico.

Objeto del contrato: El contratista se comprometerá con el municipio de Neiva de manera autónoma e independiente a prestar sus servicios profesionales como abogado especialista externo para la asesoría Jurídica para el Macroproyecto Parque Ronda Río Las Ceibas.

'Alcaldía Municipal de Neiva, oficina de Contratación.

${ }^{10}$ Alcaldía Municipal de Neiva, oficina de Contratación. 
Contratista: Pedro Felipe Andrade Monje.

Objeto: Prestar sus servicios como abogado especialista externo para la asesoría Jurídica para el Macroproyecto Parque Ronda Río Las Ceibas. Valor del contrato:

$\$ 15^{\prime} 260.000$

Valor Total:

\subsection{Contrato: No.503-2005}

\section{Programa: Saneamiento básico.}

Objeto del contrato: Construcción de obras de control y protección en el río las Ceibas paralelo a la vía que conduce de Neiva a Platanillal en el $\mathrm{K}$ $17+300$ de la vereda Platanillal y Ceibas afuera.

Actividades a realizar en el contrato: Desvío de la fuente y manejo del rio, excavaciones a todo factor y construcción de espolones y muros de contención de Gaviones.

Objeto: El contratista se obliga para con el Municipio de Neiva a realizar por el sistema de precios unitarios la construcción de obras de control y protección en el rio las Ceibas paralelo a la vía que conduce de Neiva a Platanillal en el K 17+300 de la vereda Platanillaly Ceibas afuera.

Contratista: Iván Javier Puentes Rodríguez.

Valor inicial del contrato:

$\$ 15^{\prime} 228.750$

Valor final del contrato:

$\$ 15^{\prime} 228.650$

\subsection{Contrato: $\mathrm{No} .298-2005^{12}$}

Programa: Saneamiento básico.

Estudio de conveniencia y oportunidad: Se pretende apoyar a la recuperación urbanística, ambiental del sector y adicionalmente la reubicación de las familias asentadas en estas aéreas (compra de mejoras y/o ejecución de proyectos de viviendas para su reubicación.). Identificando las mejoras ubicadas en las zonas de influencia del Macroproyecto Parque Ronda Río

Las Ceibas, determinando las aéreas de terreno que pueden ser recuperadas para el desarrollo de obras del Parque.

Contratista: John Jairo Gamboa Rodríguez.

Objeto: El contratista se obliga para con el Municipio de manera autónoma e independiente a prestar los servicios de Cadenero Auxiliar para apoyo a la comisión de topografía en el desarrollo del proyecto parque ronda rio las ceibas del municipio de Neiva.

Valor del contrato:

$\$ 4 ' 200.000$

Valor contrato adicional:

$\$ 2^{\prime} 100.000$

ValorTotal:

$\$ 6^{\prime} 300.000$

3.5. Proyecto "protección y recuperación de la cuenca río las ceibas-municipio de Neiva. "Parque ronda río las Ceibas""

Autor: OLGAPATRICIAGONZALEZ

\section{Contratista:LOPEZ RIVERATEOFILA}

Escrituras: 2885 de 2005, 1307 de 2005, 1139 de 2005

Pago: compra de predio No. 010200010002015 de la calle 25a $1 \mathrm{f}-83$ del Barrio Ceibitas para el macroproyecto parque ronda Río las Ceibas. EI costo total del proyecto es de $\$ 28.330$ ' 378.326 de los cuales se solicita financiación por parte de la Nación de \$24.500'000.000.

Descripción del Problema o Necesidad: Se presentan inundaciones en la época de invierno, desmejoramiento en la calidad del agua $y$ disminución del cauce, deterioro ambiental y paisajístico del río.

Objetivo General del Proyecto: Recuperar y manejar la Cuenca del río las Ceibas de la parte urbana mediante obras de manejo para zonas ambientales críticas, manejo integral de la cuenca y cultura ambiental.

${ }^{11}$ Alcaldía Municipal de Neiva, oficina de Contratación.

${ }^{12}$ Alcaldía Municipal de Neiva, oficina de Contratación.

${ }^{13}$ Alcaldía Municipal de Neiva-Secretaria de Hacienda Municipal. 
Descripción del Proyecto: Se adecuará el cauce del río y se construirán jarrillones para la estabilización del suelo. Las obras a ejecutarse son: preliminares ylas obras de estabilización.

Descripción de la Situación actual: Se presentan inundaciones en la época del inviemo, desmejoramiento en la calidad de agua y disminución del caudal, deterioro ambiental y paisajístico del río.

Situación Esperada: Mejoramiento ambiental y urbanístico del entorno del río las Ceibas.

\subsection{Contrato de obra publica No 604 de $2006^{14}$}

Objeto: Contrato de obra pública por el sistema de precios unitarios para realizar las reparaciones locativas en el campamento destinado a la vigilancia y control de la reserva forestal del río las ceibas ubicada en la vereda LA PLATA zona rural del municipio de Neiva departamento del Huila.

Valor: veintitrés millones novecientos noventa y nueve mil trescientos setenta y cinco mil pesos (\$23.999.375.00).

Forma de Pago: El municipio de Neiva, otorgará anticipo del $50 \%$ del valor del contrato respectivo, una vez firmada el Acta de iniciación y constituirá las garantías necesanias para el cumplimiento del objeto contractual. El $50 \%$ restante se cancelará en pagos parciales de acuerdo al avance de la obra, descontando proporcionalmente el anticipo, previa certificación del interventor del contrato y previo a la presentación de los certificados a satisfacción por la comunidad.

Plazo de Ejecución: Dos meses.

Plazo de Vigencia: Siete meses.

Liquidación: Dentro de los cuatro (4) meses al vencimiento del término de ejecución contractual, de mutuo acuerdo o de manera unilateral si fuera el caso.

\subsection{Contrato No. 698 DE $2006^{15}$}

Objeto: Contratar el suministro y la instalación de 105 tanques sépticos prefabricados, (polietileno) y con capacidad de 1000 litros, para el tratamiento de las aguas servidas 0 aguas negras en la zona rural del municipio de Neiva.

Contratista: Edna Cristina Guzmán Ramírez

Población Beneficiada: Municipio de Neiva

(zona rural).

Valor Total: $\$ 111.327 .056 .00$

En las instalaciones de la Dirección de asistencia técnica y rural y medio ambiente DATMA se reunieron la Ing. GUERLY NELLY SERRANO, interventor y la señora EDNACRISTINA GUZMAN RAMIREZ, contratista, con el objeto de realizar el acta de recibo final del contrato de suministro, después de haber recibido y verificado lo estipulado y contratado el día 11 de Septiembre de 2007.

\subsection{Contrato de Obra Pública No669 DE 2006 ${ }^{16}$}

Objeto: Desarrollar proceso de recuperación en cuatro fenómenos erosivos con procesos biológicos, en la vereda LA FLORIDA, corregimiento deAipecito del municipio de Neiva.

\section{Contratista:}

CORPORACIONAMBIENTAL CUCHIYUYO

VALOR: $\$ 24.000 .000 .00$

PLAZO:Tres meses

\subsection{Contrato No 178 de $2.007^{17}$}

Objeto: Llevar a cabo la coordinación del seguimiento, control y revisión de los predios de la reserva forestal del río las Ceibas propiedad del municipio de Neiva. Así mismo realizar recorridos para monitorear del río las Ceibas y sus principales afluentes y el acompañamiento a personal de campo, lo anterior en procura de evitar

${ }^{4}$ ALCALDIA MUNICIPAL DE NEIVA-TESORERIA MUNICIPAL-Contratos sobre Rondas Rio Las Ceibas.2005-2007

${ }^{15}$ ALCALDIA MUNICIPALDE NEIVA-TESORERIA MUNICIPAL-Contratos sobre Rondas Rio Las Ceibas.2005-2007

${ }^{16}$ ALCALDIA MUNICIPAL DE NEIVA-TESORERIA MUNICIPAL-Contratos sobre Rondas Rio Las Ceibas.2005-2007

${ }^{17}$ Alcaldía Municipal de Neiva, oficina de Contratación. 
y/o denuncias hechos y accidentes que afecten ambientalmente esta zona de reserva forestal del río las Ceibas, surtidora del acueducto municipal de la ciudad.

Contratista: Álvaro Romero Barreiro

Valor: $\$ 9.990 .000 .00$

\section{SITUACIÓN DE}

\section{IA CUENCA}

La situación actual de la Cuenca de acuerdo a los contratos, encuestas, entrevistas, asesorías y visitas a las diferentes entidades, junto con todos los estudios realizados nos dice que aunque se ha invertido en la Cuenca y se ha tratado de salvar esta fuente abastecedora de agua que surte a toda la ciudad de Neiva, no se ha invertido en problemas primordiales, como lo es la evacuación de algunas personas que se encuentran en las veredas de Motilón y la Plata, y así también salvar esta vía que comunica al Huila con el Caquetá, además no se han tomado medidas de protección especial frente al problema y no se ha ejercido un control riguroso frente a las quemas, la deforestación, las avalanchas, las cuales son continuas y aunque se le ha hecho inversión, el problema sigue latente porque no se ha canalizado lo principal, sino que se han resuelto problemas secundarios por falta de ver la realidad en la que se encuentra nuestra Cuenca, por tanto se debe retomar un nuevo plan que resuelva lo que se relaciona con la realidad de SALVAR la Cuenca que se encuentra en grave peligro y así lograr encaminar la administración a la solución del problema principal, al que consideramos que es la compra de los predios y la evacuación definitiva de sus habitantes.

\section{CONCLUSIONES}

1. Los problemas que afronta la Cuenca del Río las Ceibas se basan en que aunque está detectado el problema que la aqueja no se está invirtiendo el dinero en lo que realmente es la necesidad primordial sino en otras actividades secundarias que no son tan relevantes para la Preservación y Conservación de la Cuenca.
2. La poca colaboración de la ciudadanía que insiste en la tala de árboles, quemas, creación de basureros y demás.

3. No hay un verdadero interés de común acuerdo entre el Estado y la población, ya que el Estado debería lograr que lagente que habita las zonas de alto riesgo evacue y éstas personas a la vez prestar una colaboración inmediata ya que la vida de ellas corre peligro por los constantes deslizamientos que la cuenca presenta sobre todo en tiempo de invierno.

4. Negligencia por parte del Estado y la comunidad en la preservación y conservación de la Cuenca, ya que la necesidad de salvarla es apremiante, $y$ las personas de alli vecinas sólo se preocupan en su supervivencia sin importar el riesgo de que muera la Cuenca y el Estado no se apresura a tomar medidas eficientes en lo más importante, que es impedir a toda costa la quema y tala de árboles y en invertir el dinero lo más urgentemente posible en la recuperación de éstas y no en obras de mamposteria.

5. Las obras construidas, especialmente los gaviones, están en muy mal estado por las constantes avalanchas que presenta la cuenca, razón por la cual no es acertado la construcción de este tipo de obras.

6. Los baños, albercas y pozos sanitarios construidos ya se encuentran totalmente deteriorados en tampoco tiempo de estrenarlos, lo que obliga a la comunidad de sectores desde PLATANILLAL hasta LA PLATA a usar la Cuenca como lugar donde se les diera un tratamiento especial.

Las personas contratadas, aunque han tratado de hacer una buena labor y de cumplir el objeto del contrato, no ha tenido muy bien en claro el gran problema que afronta la Cuenca y que de seguir así sólo se logrará que cada día el estado de ésta sea más deprimente, pues las obras no suplen la necesidad, porque los gaviones hechos son muy pobres, pocos y escasos para soportar la magnitud de la cuenca en tiempo de lluvia.

7. El peligro en el que también se encuentra la vía principal a causa de los deslizamientos es grande, pues cada día el espacio que separa la carretera de la cuenca es más pequeño. 
8. El dinero invertido no ha sido el mejor utilizado, pues las obras hechas, como los gaviones se encuentran en muy mal estado, y no suplen la necesidad urgente de salvar en el menor tiempo posible la Cuenca del Río las Ceibas.

\section{BIBLIOGRAFÍA}

- ACERO, S. JOSÉ. Plan de recuperación y manejo de la cuenca del río las Ceibas, cuantificación técnico-económica del plan. Neiva, diciembre, 1991.

- ALCALDIA MUNICIPAL DE NEIVA - CONCEJO MUNICIPAL, CM, proyecto en el cual se divide en corregimientos el territorio de la zona rural el municipio de Neiva.

- ALCALDIA MUNICIPAL DE NEIVA - DEPARTAMENTO DE PLANEACION MUNICIPAL, PLAN DE ORDENAMIENTO MUNICIPAL, POT., Matriz prioridad de acciones -corregimiento oriente. Neiva, 2000 a 2010 Alcaldía Municipal de Neiva-Departamento.

- Planeación Municipal, Plan de Ordenamiento Territorial Municipio Neiva 2000-2010.

- ALCALDIAMUNICIPAL DE NEIVA-OFICINA JURIDICA. Contratos hechos para la recuperación de la Cuenca. 2005-2007.

- ALCALDIA MUNICIPAL DE NEIVA-TESORERIA MUNICIPAL- Contratos sobre RONDAS RIO LAS CEIBAS.2005-2007.

- ALCALDIA MUNICIPAL DE NEIVA-ALMACEN- DATMA - SECRETARIA DE HACIENDA MUNICIPAL SECRETARIADE INVIAS -Contratos RECUPERACION RIOLASCEIBAS 2005-2007

- CONTRALORIA DEPARTAMENTAL DEL HUILA. Estudio anual sobre del estado de los recursos naturales y medio ambiente del Río las Ceibas. Neiva, 2000 .REVISTASOS DE2000.

- CONTRALORIA DEPARTAMENTAL DEL HUILA. Ecosistemas estratégicos en la Subcuenca del Río las Ceibas. Neiva 2000.

- CONTRALORIA MUNICIPAL DE NEIVA, INFORME MEDIO AMBIENTE, evaluación de la gestión ambiental del municipio de Neiva en la vigencia fiscal del 2003. Neiva, julio de 2004.

- CORPORACIÓN AUTONOMA REGIONAL DEL ALTO MAGDALENA, CAM, evaluación, priorización, cuantificación y recomendación de obras para el control de erosión visible de la Cuenca Río las Ceibas CORPORACION SEMBRAR PROGRESO Y PAZ, CSPP, plan de inversión para la protección y recuperación de la cuenca del río las ceibas. Neiva, septiembre de 2003.

- EMPRESAS PÚBLICAS DE NEIVA-Contratos de la Cuenca entre 2005-2007

- GOBERNACION DEL HUILA, SECRETARIA DE DESARROLLO AGROPECUARIO Y MINERO, calidad fisicoquímica y bacteriológica del agua del Río las Ceibas, USCO facultad de ingeniería. Neiva 1997.

- GOBERNACION DEL HUILA, SECRETARIA DE AGRICULTURA Y MINERIA, anuario estadístico agrícola, pecuario, piscicola, forestal y minero. Neiva 2004.

- INSTITUTO NACIONAL DE LOS RECURSOS NATURALES RENOVABLES Y DEL MEDIO AMBIENTE, INDERENA. Síntesis de la problemática y propuestas de acciones en cuenca del Río las Ceibas. Neiva,Abril de 1989.

- INSTITUTO NACIONAL DE LOS RECURSOS NATURALES RENOVABLES Y DEL MEDIO AMBIENTE, INSTITUTO DE INVESTIGACIONES DE RECURSOS BIOLOGICOSALEXANDER VON HUMBOLT, programa de investigación en política y legalizaron de indicadores de seguimiento de la política de biodiversidad, programa de biología de la conservación. Bogotá, 30 de octubre de 2002. 
- MINISTERIO DEL MEDIO AMBIENTE INSTITUTO NACIONAL DE LOS RECURSOS NATURALES RENOVABLES Y DELAMBIENTE, INDERA, programa de protección y manejo de la cobertura vegetal de la Cuenca del Río Las Ceibas.

- MINISTERIO DEL MEDIO AMBIENTE INSTITUTO NACIONAL DE LOS RECURSOS NATURALES RENOVABLES Y DELAMBIENTE, INDERA, importancia y legado del proyecto cuenca alto Magdalena y sus centros experimentales, memorias 1982-1994. Bogotá, mayo de 1995.

- PROYECTO CUENCADELALTOMAGDALENACORPORACIÓNAUTONOMA

- REGIONALDELALTO MAGDALENA, PROCAM, Plan de Comercialización Agropecuaria Cuenca Ríos las Ceibas, Yaguará y Combeima. Bogotá, julio de 1986

- UNIVERSIDAD SURCOLOMBIANA CENTRO DE INVESTIGACIONES Y DESARROLLO CIENTIFICO, USCO, CIDEC, monografía de la subcuenca del Río las Ceibas. Recopilación de información Neiva 1990.

- UNIVERSIDAD SURCOLOMBIANA, USCO, FACULTAD DE INGENIERIA AMBIENTAL, valoración económica aproximada de los costos ambientales de la cuenca del Río las Ceibas en el Municipio de Neiva-Huila de 1999. 